Article

\title{
Sensitivity and Selectivity of Two Commercially Available Media for Legionella spp. Recovery from Environmental Water Samples
}

\author{
Savina Ditommaso*(D, Monica Giacomuzzi, Gabriele Memoli, Jacopo Garlasco $\mathbb{D}$ \\ and Carla M. Zotti \\ Department of Public Health and Pediatrics, University of Turin, 10126 Turin, Italy; \\ monica.giacomuzzi@unito.it (M.G.); gabriele.memoli@unito.it (G.M.); jacopo.garlasco@unito.it (J.G.); \\ carla.zotti@unito.it (C.M.Z.) \\ * Correspondence: savina.ditommaso@unito.it; Tel.: +39-0116705841; Fax: +39-0116705881
}

Received: 11 May 2020; Accepted: 22 June 2020; Published: 29 June 2020

\begin{abstract}
The quality control of culture media used for Legionella spp. isolation and enumeration is paramount to achieve a satisfactory degree of comparability among water testing results from different laboratories. Here, we report on a comparative assessment of the sensitivity and selectivity of MWY and BCYE $\alpha$ media supplied by two different manufacturers (i.e., Xebios Diagnostics $\mathrm{GmbH}$ and Oxoid Ltd) for the detection of Legionella spp. from environmental water samples. Even though our analysis showed an excellent agreement between the recovery rates of the four media tested $(90.5 \%)$, the quantitative recovery of Legionella spp. colonies using Xebios media was significantly greater than that achieved by Oxoid media $(P=0.0054)$. Furthermore, the sensitivity of detection was significantly higher when samples were plated on MWY Xebios agar $(P=0.0442)$, while the selectivity of MWY appeared to be the same regardless of the manufacturer. Furthermore, MWYXebios agar favored the growth of much larger colonies compared to those observed on MWYOxoid agar. Finally, MWYXebios medium enhanced the recovery of non-pneumophila Legionella species. Collectively, our findings demonstrate that quality control is crucial to ensure high selectivity and sensitivity of the culture media used for the detection and enumeration of Legionella spp. from environmental water resources. As water remediation measures strictly depend on Legionella spp. recovery, culture protocol standardization, as well as quality control of the culture media, is essential to achieve intra- and interlaboratory reproducibility and accuracy.
\end{abstract}

Keywords: Legionella spp.; culture media; environmental monitoring; quality control

\section{Introduction}

The most common protocol for environmental surveillance of Legionella spp. involves the use of buffered charcoal yeast extract agar enriched with $1 \mathrm{~g} / \mathrm{L}$ alpha-ketoglutarate agar $(\mathrm{BCYE} \alpha)$. Although this method has a proven record of effectively isolating and enumerating Legionella species from environmental or clinical specimens, its sensitivity and selectivity are often hampered by the presence of contaminating flora in the water samples, which may influence the final count of Legionella spp. due to overgrowth or inhibition. Therefore, culture on selective agar media-i.e., BCYE $\alpha$ supplemented with glycine, vancomycin, polymyxin B, cycloheximide (GVPC) or with glycine, polymyxin B, vancomycin, anisomycin, bromothymol blue, and bromocresol purple (MWY) - that is capable of inhibiting most non-Legionellaceae bacteria is the preferred solution to isolate Legionella spp. from environmental specimens [1-3].

Given that the recovery of Legionella spp. strictly depends on the type of agar being used, quality-assured culture media for water testing are key to consumer safety. However, the overall 
performance of commercially available nonselective (BCYE $\alpha$ ) and selective (MWY and GVPC) media for Legionella spp. isolation has only been partially addressed. In this regard, the last studies on the quality assurance of these media date back to 2004 [4] and 2010 [5].

According to the literature, these types of media are very difficult to prepare, store, and test as minor differences in $\mathrm{pH}$, cation content, and agar composition can heavily influence growth rates, plating efficiency, and colony formation [6-8]. Thus, the quality control of culture media used for Legionella spp. detection is now, more than ever, essential to achieve a satisfactory degree of comparability among water-testing results from different laboratories.

For over two decades, our group has been conducting Legionella spp. testing in numerous hospitals and health facilities in the Piedmont region of Italy. Furthermore, since 2007, our laboratory has been a permanent member of the External Quality Assessment (EQA) scheme for Legionella spp. isolation from water, which relies on the use of the BCYE $\alpha$ and MWY media manufactured by Oxoid Ltd. (Basingstoke, UK), with satisfactory z-scores of performance throughout. In particular, by conducting parallel seeding experiments, we have previously found that BCYE $\alpha$ allows a higher yield and recovery rate of Legionella spp. positive samples (93\%) compared to that obtained with MWY (78\%). Based on these findings, we were the first in 2011 [9] to recommend the use of BCYE $\alpha$ as a nonselective medium, in addition to MWY or GVPC, for optimal detection of Legionella spp. in environmental water samples. The combined use of one selective and one nonselective media for improved Legionella spp. detection was later on incorporated in the second edition of ISO 11731 [10].

Besides the recovery rate, we have also been actively involved in evaluating the sensitivity and selectivity of nonselective vs. selective media for the isolation and enumeration of Legionella spp., an investigation that has been more recently extended to $\mathrm{BCYE} \alpha-\mathrm{AB}$ media (Ditommaso et al., unpublished data).

In this study, we report on a comparative assessment between the sensitivity and selectivity of the aforementioned media from Oxoid Ltd. (Basingstoke, UK) and the corresponding media manufactured by Xebios Diagnostics GmbH (Düsseldorf, Germany) for the detection of Legionella spp. from environmental water samples.

\section{Results}

\subsection{Overall Results}

A total of 148 water samples were cultured on MWY and BCYE $\alpha$ agar produced by two different manufacturers (i.e., Xebios and Oxoid). Bacteria were isolated from 70/148 samples (47.3\%) using either type of medium, showing comparable levels of Legionella spp. detection. Specifically, we detected $64(43.2 \%)$ and $62(41.9 \%)$ positive cultures using Xebios and Oxoid mediums, respectively, with excellent agreement between the two brands $(90.5 \%$; $\kappa=0.807)$.

According to the Wilcoxon test analysis performed on 148 results obtained with each medium, the detection sensitivity increased when the samples were plated on Xebios medium $(P=0.0054$, median difference between log-counts: $\left.\Delta=0.192, \mathrm{CI}_{95 \%}: 0.055,0.394\right)$. Consistently, the 56 concordant positive samples also displayed the highest counts on Xebios agar plates $(P=0.0006, \Delta=0.159$, $\left.\mathrm{CI}_{95 \%}: 0.068,0.295\right)$.

\subsection{Results Relative to Medium Type}

To further investigate the differences in the recovery of Legionella spp., we assessed the sensitivity and selectivity of Oxoid vs. Xebios alpha-ketoglutarate agar (BCYE $\alpha$ ) and bromocresol purple (MWY) in detecting Legionella spp. from 148 environmental water samples (Table 1). 
Table 1. Relative sensitivity and selectivity of BCYE $\alpha$ and MWY media from Xebios or Oxoid for Legionella spp. isolation.

\begin{tabular}{ccccc}
\hline & BCYE $\alpha^{\text {Oxoid }}$ & MWY Oxoid & BCYE $\alpha^{\text {Xebios }}$ & MWY Xebios \\
\hline Number (\%) plates growing Legionella & $44(29.7)$ & $52(35.1)$ & $49(33.1)$ & $62(41.9)$ \\
Sensitivity $^{\text {a }}$ & $(63)$ & $(74)$ & $(70)$ & $(78)$ \\
$\begin{array}{c}\text { Number (\%) plates growing } \\
\text { microorganism other than Legionella } \\
\text { Selectivity }\end{array}$ & $21(14.2)$ & $70(47.3)$ & $18(12.2)$ & $70(47.3)$ \\
\end{tabular}

a Sensitivity was calculated by comparing the number of positive plates for a given medium with the cumulative yield of Legionella spp. from all four media $(n=70) .{ }^{\mathbf{b}}$ Selectivity for each media was defined as the number of plates suppressing the growth of organisms that were not Legionella spp. over the total number of plates $(n=148)$.

The detection sensitivity was significantly higher in samples plated on MWY $\mathrm{Y}^{\mathrm{Xebios}}$ agar compared to that of samples grown on MWYOxoid agar (McNemar test: $P=0.0042)$. No difference was found between $B C Y E \alpha^{\text {Oxoid }}$ and BCYE $\alpha^{\text {Xebios }}$ media (McNemar test: $P=0.03588$ ); no difference in suppressing non-Legionella bacteria (i.e., selectivity) was found between the two brands of MWY agar.

A Wilcoxon signed-rank test comparison (Table 2) revealed a significant difference in the number of Legionella spp. colonies, not only between BCYE $\alpha^{\mathrm{Xebios}}$ and $\mathrm{MWY} \mathrm{Y}^{\mathrm{Xebios}}$ media but also between MWYOxoid and MWYXebios media. In either case, the recovery of Legionella spp. was significantly higher when the samples were plated on MWY ${ }^{\text {Xebios }}$ agar.

Table 2. Wilcoxon signed-rank test analysis of Legionella spp. counts (CFU/L) obtained with four different types of mediums.

\begin{tabular}{ccccc}
\hline & Xebios Media vs. Oxoid Media & $\boldsymbol{P}$-Value & $\boldsymbol{\Delta}\left(\mathbf{C I}_{\mathbf{9 5} \%}\right)$ \\
\hline (a) & BCYE $\alpha$ Oxoid & MWY Oxoid & 0.476 & \\
(b) & BCYE $\alpha$ Xebios & MWY Xebios & 0.0005 & $\Delta=0.515\left(\mathrm{CI}_{95 \%}: 0.182,1.023\right)[\mathrm{MWY}>\mathrm{BCYE}]$ \\
(c) & BCYE $\alpha$ Oxoid & BCYE $\alpha$ Xebios & 0.0905 & \\
(d) & MWY Oxoid & MWY Xebios & 0.0014 & $\Delta=0.618\left(\mathrm{CI}_{95 \%}: 0.268,0.981\right)[$ Xebios $>$ Oxoid $]$ \\
\hline
\end{tabular}

Comparison between corresponding media from the two companies $(a, b)$ and between different types of medium from each company $(\mathrm{c}, \mathrm{d}) . \Delta$ is the median of the differences of log-counts, where the left column is the reference.

All data were disaggregated according to medium and manufacturer. Table 3 classifies the samples according to the presence or absence of Legionella spp. in MWY and BCYE media from Oxoid (Table 3a) or Xebios (Table 3b). There is a greater agreement between the two Xebios media (47/49) in comparison with that between the two Oxoid media (34/44).

Table 3. Assessment of Legionella spp. recovery according to culture medium and manufacturer.

\begin{tabular}{|c|c|c|c|c|c|c|c|c|c|c|c|c|}
\hline & \multicolumn{4}{|c|}{ (a) OXOID } & \multicolumn{4}{|c|}{ (b) XEBIOS } & \multicolumn{4}{|c|}{$\begin{array}{l}\text { (c) EXPECTED XEBIOS } \\
\text { [from (a) with Deming-Stephan Method] }\end{array}$} \\
\hline & \multicolumn{4}{|c|}{ BCYE $\alpha$} & \multicolumn{4}{|c|}{ BCYE $\alpha$} & \multicolumn{4}{|c|}{ ВСYE $\alpha$} \\
\hline \multirow{4}{*}{ MWY } & & $\begin{array}{l}\text { Pos } \\
\text { (n) }\end{array}$ & $\begin{array}{c}\text { Neg } \\
\text { (n) }\end{array}$ & Total & & $\begin{array}{l}\text { Pos } \\
\text { (n) }\end{array}$ & $\begin{array}{c}\text { Neg } \\
\text { (n) }\end{array}$ & Total & & $\begin{array}{l}\text { Pos } \\
\text { (n) }\end{array}$ & $\begin{array}{c}\text { Neg } \\
(n)\end{array}$ & Total \\
\hline & $\begin{array}{c}\text { Pos } \\
\text { (n) }\end{array}$ & 34 & 18 & 52 & $\begin{array}{c}\text { Pos } \\
\text { (n) }\end{array}$ & 47 & 15 & 62 & $\begin{array}{l}\text { Pos } \\
\text { (n) }\end{array}$ & 40.22 & 21.78 & 62 \\
\hline & $\begin{array}{c}\text { Neg } \\
\text { (n) }\end{array}$ & 10 & 86 & 96 & $\begin{array}{c}\text { Neg } \\
(n)\end{array}$ & 2 & 84 & 86 & $\begin{array}{l}\text { Neg } \\
(n)\end{array}$ & 8.78 & 77.22 & 86 \\
\hline & Total & 44 & 104 & 148 & Total & 49 & 99 & 148 & Total & 49 & 99 & 148 \\
\hline
\end{tabular}

Pearson's chi-squared test comparison between Oxoid and Xebios results, after a Deming-Stephan adjustment, revealed a statistically significant difference between the two brands $\left(\chi^{2}=9.0824\right.$, $P=0.0026$; Table 3c). 
The number of Legionella spp. colonies (CFU/L) growing or not on BCYE $\alpha$ and MWY is shown in Tables 4 and 5.

Table 4. Number of Legionella spp. colonies (CFU/L) appearing on different plates: BCYE $\alpha$ positive results vs. MWY negative results.

\begin{tabular}{|c|c|c|c|c|c|c|c|c|c|c|c|}
\hline \multicolumn{6}{|c|}{ Oxoid } & \multicolumn{6}{|c|}{ Xebios } \\
\hline Samples & BCYE $\alpha$ & MWY & L. spp. & $\begin{array}{c}\text { B. Flora } \\
\text { on } \\
\text { BCYE } \alpha\end{array}$ & $\begin{array}{l}\text { B. Flora } \\
\text { on } \\
\text { MWY }\end{array}$ & Samples & ВСYЕ $\alpha$ & MWY & L. spp. & $\begin{array}{l}\text { B. Flora } \\
\text { on } \\
\text { BCYE } \alpha\end{array}$ & $\begin{array}{c}\text { B. Flora } \\
\text { on } \\
\text { MWY }\end{array}$ \\
\hline 1 & 50 & 0 & Lp3 & $1+$ & 0 & 1 & 50 & 0 & Lp3 & 0 & I \\
\hline 2 & 50 & 0 & Lp1 & $1+$ & 0 & 2 & 500 & 0 & Lp1 & $1+$ & I \\
\hline 3 & 150 & 0 & Lp6 & $1+$ & 0 & & & & & & \\
\hline 4 & 200 & 0 & L.spp & 0 & 0 & & & & & & \\
\hline 5 & 400 & 0 & L.spp & 0 & 0 & & & & & & \\
\hline 6 & 600 & 0 & L.spp & $1+$ & 0 & & & & & & \\
\hline 7 & 1050 & 0 & L.spp & 0 & 0 & & & & & & \\
\hline 8 & 2200 & 0 & L.spp & $2+$ & 0 & & & & & & \\
\hline 9 & 2350 & 0 & L.spp & 0 & 0 & & & & & & \\
\hline 10 & 3400 & 0 & L.spp & 0 & 0 & & & & & & \\
\hline
\end{tabular}

Background (B.) flora was measured by semiquantitative counting: four categories were determined according to the visual density of colonies spread onto the plate, where zero is no background flora and $3+$ is massive contamination (see supplementary materials). Lp $1=$ L. pneumophila serogroups $1 ; \mathrm{Lp} 3=$ L. pneumophila serogroups 3 ;

Lp 6 = L. pneumophila serogroups 6; L.spp. = Legionella spp. non-pneumophila.

Table 5. Number of Legionella spp. colonies (CFU/L) appearing on different plates: BCYE $\alpha$ negative results vs. MWY positive results.

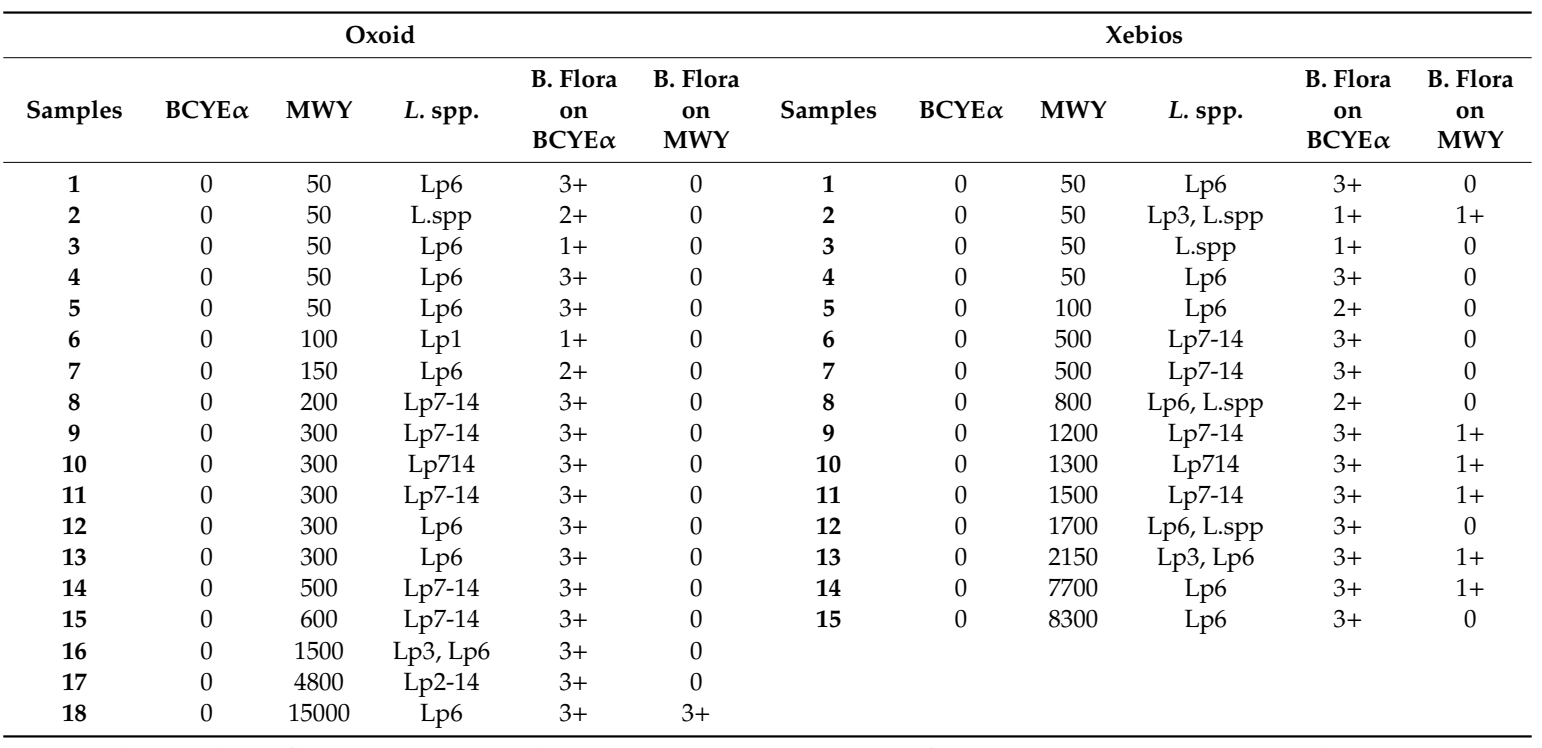

Background (B.) flora was measured by semiquantitative counting: four categories were determined according to the visual density of colonies spread onto the plate, where zero is no background flora and 3+ is massive contamination (see supplementary materials). Lp $1=$ L. pneumophila serogroups 1 ; Lp $3=$ L. pneumophila serogroups 3; Lp $6=$ L. pneumophila serogroups 6; Lp 2-14 $=$ L. pneumophila serogroups 2-14; Lp 7-14= L. pneumophila serogroups 7-14; L.spp = Legionella spp. non-pneumophila .

The observation that some water samples were Legionella spp. positive on BCYE $\alpha$ medium but negative on MWY agar (7/10 cultures, samples 4-10, Table 4) indicates that the use of selective medium can affect the recovery of non-Legionella pneumophila species. Cell concentrations from these water samples ranged from $2.0 \times 10^{2}$ to $3.4 \times 10^{3} \mathrm{CFU} / \mathrm{L}$. These results only refer to samples cultured on Oxoid media (Table 4).

Table 5 shows that high levels of background flora can challenge the results: on BCYE $\alpha$ agar, the results were affected by the presence of concomitant background flora that prevented the growth or masked the observation of Legionella colonies in $\mathrm{BCYE} \alpha$ agar from both manufacturers. No qualitative 
data are available for these aquatic bacteria. Contaminating non-Legionella bacteria were rarely recovered on MWY agar from either manufacturer.

The influence of the media on the detection time was also assessed. Typically, antibiotics added to the selective media suppress the accompanying flora but at the same time slow down the growth of the target organism. The average colony size of Legionella spp. cultured on Xebios media was greater than that of Legionella spp. plated on Oxoid medium, especially for bacteria plated onto MWY agar. With regard to colony count, the number of Legionella spp. was higher on MWY ${ }^{\mathrm{Xebios}}$ agar compared to MWY ${ }^{\text {Oxoid }}$. As for the Oxoid media, even though the supplemented antibiotics suppressed the accompanying flora, it slowed down excessively the growth of the target organisms. No particular differences in colony size or count were observed between the two brands of BCYE $\alpha$ agar, as already shown in Table 2 (see also the supplementary materials).

\section{Discussion}

Several factors may hinder exact Legionella spp. quantification in environmental samples: (i) differences in the polycarbonate membrane characteristics (e.g., pore size, batches, fragility, crinkling and electrostatic interactions), (ii) different washing procedures to remove trapped bacteria from the membrane (e.g., shaker/vortex, ultrasound, finger and thumb scraping, or heat or acid treatment), which favor the detection of the microorganism but at the same time may reduce its concentration, and (iii) the choice of the culture medium [11-14].

As for the latter, the parameters affecting its quality are the following: (1) type and quantity of nutrients, (2) redox potential (Eh)—both after preparation and during incubation, (3) initial $\mathrm{pH}$ and buffering capacity, (4) water activity, and (5) type and activity of the antimicrobial agents-these can either be supplemented, already be present in the medium components, or accidentally form due to preparation errors, such as excessive heating $[6,15]$. Further evidence has highlighted several other deficiencies of those selective media that rely on a delicate balance between productive and selective mechanisms $[4,8,16,17]$.

The quality of culture media has a dramatic effect on Legionella spp. recovery and counts. To evaluate the contribution of culture media, we checked relative recoveries of Legionella spp. from 148 environmental water samples because the response obtained from media plated with collection strains (i.e., quality control protocol) may vary when wild bacterial strains are present. It is known, for example, that virulent L. pneumophila are especially salt-sensitive, and that spontaneous mutations in stock strains may result in salt resistance [18]. Consequently, testing culture media using stock strains of bacteria may not always be a valid approach [5].

Although several different medium formulations are routinely used to detect Legionella spp. from environmental samples [10], there is paucity of studies assessing and comparing their abilities in growing Legionella spp. $[4,5,9]$. Since the quality of the culture medium strongly influences Legionella spp. detection and enumeration due to the presence of contaminating flora, here, we have assessed the recovery rates of Legionella spp. from hospital water samples using two different brands (i.e., Xebios and Oxoid) of either nonselective BCYE $\alpha$ or selective MWY medium.

Our analysis shows an excellent agreement between the recovery rates of the media from both companies (90.5\%). Nonetheless, the quantitative recovery of Legionella colonies using Xebios media is significantly greater than that achieved by Oxoid media. Furthermore, the sensitivity of detection is significantly higher when samples are plated on MWY Xebios agar, while the selectivity of MWY appears to be the same regardless of the manufacturer. Moreover, there is a greater agreement between the two Xebios media compared to that between the two Oxoid media. Additionally, differences in colony size were apparent for the different agars (see supplementary materials). Specifically, the MWY ${ }^{\text {Xebios }}$ agar favored the growth of much larger colonies compared to MWYOxoid, and enhanced the recovery of non-Legionella pneumophila species.

As we used four different batches over a one-year period of study, it is highly unlikely that batch-to-batch variability may have played a role in the performance differences that we observed. 
We hypothesize that other factors such as the presence in the medium of toxic compounds (e.g., metals), growth-promoting factors, or high gel strengths may have influenced the growth and colony size on different types of medium, as previously shown for L. pneumophila on BCYE $\alpha$ agar [19].

Collectively, these results highlight significant differences between the performances of media from different manufacturers. Despite generating the same number of positive cultures, the Xebios media generally yielded greater numbers of Legionella spp. and larger colony sizes, allowing easier detection. Thus, the use of Xebios culture media is indicated to achieve the highest sensitivity and selectivity when detecting environmentally sampled L. pneumophila.

\section{Methods}

\subsection{Water Samples}

Media were tested using environmental samples obtained from hospital building waterlines. One liter of sample was collected from each site in sterile one-liter plastic bottles. A sodium thiosulphate $(100 \mathrm{mg} / \mathrm{L})$ solution was added to the samples to neutralize free chlorine in treated water supplies.

\subsection{Culture Media}

To distinguish between Legionella spp. and non-Legionella bacteria, two different media were used: $0.1 \%$ BCYE $\alpha$ agar and BCYE $\alpha$ agar supplemented with $3 \mathrm{~g} / \mathrm{L}$ glycine, 50,000 IU of polymyxin $\mathrm{B}$, $0.001 \mathrm{~g} / \mathrm{L}$ of vancomycin, $0.08 \mathrm{~g} / \mathrm{L}$ of anisomycin, $0.01 \mathrm{~g} / \mathrm{L}$ of bromothymol blue, and $0.01 \mathrm{~g} / \mathrm{L}$ of bromocresol purple (MWY). The formulations of both media conform to ISO 11731 [10].

Commercially available agar plates were purchased by Oxoid Ltd. (Basingstoke, UK) or Xebios Diagnostics GmbH (Düsseldorf, Germany). For each batch supplied, each manufacturer provided us with detailed quality control information (e.g., type of bacteria, $\mathrm{pH}$, colony morphology, selectivity, recovery and expected results). The microbiological performance test was carried out in accordance with ISO 11133:2014 requirements [20]: for BCYE $\alpha$ agar, colony count of positive strains was $\geq 70 \%$ for each inoculum (i.e., productivity); for MWY agar, colony count of positive strains was $\geq 50 \%$ for each inoculum (i.e., selectivity).

\subsection{Laboratory Procedure}

Briefly, the water samples were concentrated 100-fold by filtration through a $0.2-\mu \mathrm{m}$ polycarbonate filter (Millipore, Billerica, MA, USA). The filter membrane was aseptically placed in one of the bottom corners inside the stomacher bag and rubbed with the finger and thumb of one hand for $1 \mathrm{~min}$ with $10 \mathrm{~mL}$ Page solution ( $\mathrm{pH} 6.8$ ) to detach the bacteria. A $0.2-\mathrm{mL}$ volume of the concentrated sample was spread on duplicate plates of MWY or BCYE $\alpha$ agar. The plates were incubated at $36^{\circ} \mathrm{C}$ in a humid $2.5 \%$ $\mathrm{CO}_{2}$ chamber and examined after 3, 6, and 10 days of incubation. Suspected colonies were subcultured on blood and BCYE $\alpha$ agar.

\subsection{Identification of Legionella spp.}

The presence of background flora was measured through semiquantitative counting. According to the visual density of the colonies spread onto the plate, four categories were determined, where zero represented no background flora and $3+$ massive contamination (see supplementary materials). Colonies grown on MWY or BCYE $\alpha$ agar were subsequently identified by agglutination test (Legionella latex test; Oxoid). This test allows the separate identification of L. pneumophila Serogroup 1 and Serogroups 2 to 14 and detection of seven other species of Legionella (polyvalent). Colonies identified as L. pneumophila Serogroup 2 to 14 were further tested with Legionella agglutination latex reagents (Pro-Lab Diagnostics, Richmond Hill, Canada), which are intended for the identification of a single L. pneumophila sero group. Colonies not identified by the agglutination test were tested by polymerase chain reaction (in-house PCR) for the detection of the genus Legionella. This PCR assay utilizes specific primers to amplify the $16 \mathrm{~S}$ rRNA gene of Legionella spp. [21]. The plate showing the highest number of 
confirmed colonies was used to estimate the number of Legionella spp. in the original sample (report). Concentrations of Legionella spp. in water samples are expressed as colony forming units per liter (CFU/l). According to this method, the lower limit of detection (LOD) is $50 \mathrm{CFU} / \mathrm{L}$.

\subsection{Statistical Analysis}

Agreement between the final reports (i.e., Oxoid and Xebios) and the two media (i.e., MWY and $\mathrm{BCYE} \alpha$ ) was assessed by two-by-two contingency tables, through Cohen's $\mathrm{k}$ coefficient. Comparison between Legionella spp. counts obtained by Xebios and Oxoid media was performed by a Wilcoxon signed-rank test.

To quantify the difference between counts on different media, our analyses took into account the decimal logarithm of counts observed. The median difference was chosen as the estimator of the actual difference for each comparison, as indicated by Wilcoxon's nonparametric analysis.

The two $2 \times 2$ tables were compared using the Deming-Stephan method [22,23]. Specifically, the first table (Oxoid counts, see Table 3a) was transformed through the algorithm developed by Mosteller et al. [24] in order to obtain another table (Table 3c). These could be considered as the expected values for the Xebios table according to the results of the Oxoid count. Therefore, values in Table $4 \mathrm{c}$ were compared to the actual observed Xebios counts (Table 3b), with Pearson's chi-squared test.

The sensitivity of Legionella spp. detection was calculated by comparing the number of positive plates for a given medium with the cumulative yield of Legionella spp. from all four media. Selectivity for each method was defined as the number of plates that suppressed the growth of organisms that were not Legionella spp. Sensitivity and selectivity were compared using the exact McNemar test.

All statistical analyses were performed using the statistical software R ("stats" package, version 3.6.3) [25]. For all analyses, the level of significance was set at $\alpha=0.05$.

\section{Conclusions}

In conclusion, our data demonstrate that the quality of culture media is crucial in determining the level of Legionella spp. colonization in hospital water systems. As water remediation measures are based on quantitative Legionella spp. data obtained by culturing environmental samples, culture protocol standardization, as well as accurate quality control of the culture media, is essential to achieve intra- and interlaboratory reproducibility and accuracy.

Given the public health risk from Legionella spp., it is important that all water-testing laboratories carefully consider the following aspects: (1) there can be variability in Legionella spp. detection due to different types and brands of medium - of note, this variation can also be observed among different batches from the same supplier; (2) the medium should be purchased from a reputable company and fully validated in-house, with the inclusion of appropriate controls; (3) when switching to a different medium manufacturer, extensive validation should be performed in order to determine whether the new medium is "fit for purpose".

Supplementary Materials: The following are available online at http://www.mdpi.com/2076-0817/9/7/523/s1. File: images of plate with background flora; paired images (BCYE $\alpha$ and MWY agar by two manufacturers) obtained during incubation period of inoculated plates.

Author Contributions: Conceptualization, S.D. and M.G.; methodology, S.D. and M.G.; formal analysis, M.G. and G.M.; data curation, M.G. and J.G.; funding acquisition, C.M.Z.; writing original draft preparation, S.D. and M.G.; writing, review, and editing, S.D., M.G., J.G., and C.M.Z. All authors have read and agreed to the published version of the manuscript.

Funding: This work was supported by a grant from the Department fund ZOTC_CT_PREST_18_01, Department of Public Health and Pediatrics, University of Turin.

Conflicts of Interest: None of the authors declare to have a conflict of interest that could influence or bias the content of the paper. 


\section{References}

1. International Organization for Standardization. ISO 11731: 1998 Water Quality—Detection and Enumeration of Legionella; International Organization for Standardization: Geneva, Switzerland, April 1998.

2. Centers for Disease Control and Prevention (CDC). Legionnaires Disease Procedures Manual for Recovery | Legionnaires; CDC: Atlanta, GA, USA, 2005.

3. Health Protection Agency (HPA). Detection and Enumeration of Legionella Species by Filtration and Centrifugation; National Standards Method W 12; HPA: London, UK, 2006.

4. Lück, P.C.; Igel, L.; Helbig, J.H.; Kuhlisch, E.; Jatzwauk, L. Comparison of commercially available media for the recovery of Legionella species. Int. J. Hygiene Environ. Health 2004, 207, 589-593. [CrossRef] [PubMed]

5. Edelstein, P.H.; Edelstein, M.A.C. Comparison of the plating efficiencies and shelf lives of three different commercial buffered charcoal yeast extract media supplemented with alpha-ketoglutaric acid. J. Clin. Microbiol. 2010, 48, 1882-1883. [CrossRef] [PubMed]

6. Mossel, D.A.; Bonants-Van Laarhoven, T.M.; Ligtenberg-Merkus, A.M.; Werdler, M.E. Quality assurance of selective culture media for bacteria, moulds and yeasts: An attempt at standardization at the international level. J. Appl. Bacteriol. 1983, 54, 313-327. [CrossRef]

7. Feeley, J.C.; Gibson, R.J.; Gorman, G.W.; Langford, N.C.; Rasheed, J.K.; Mackel, D.C.; Baine, W.B. Charcoal-yeast extract agar: Primary isolation medium for Legionella pneumophila. J. Clin. Microbiol. 1979, 10, 437-441. [CrossRef] [PubMed]

8. Edelstein, P.H. Improved semiselective medium for isolation of Legionella pneumophila from contaminated clinical and environmental specimens. J. Clin. Microbiol. 1981, 14, 298-303. [CrossRef] [PubMed]

9. Ditommaso, S.; Gentile, M.; Giacomuzzi, M.; Zotti, C.M. Recovery of Legionella species from water samples using an internal method based on ISO 11731: Suggestions for revision and implementation. Diagn. Microbiol. Infect. Dis. 2011, 70, 200-206. [CrossRef] [PubMed]

10. International Organization for Standardization. ISO 11731: 2017 Water Quality_Enumeration of Legionella; International Organization for Standardization: Geneva, Switzerland, 2017.

11. Boulanger, C.A.; Edelstein, P.H. Precision and accuracy of recovery of Legionella pneumophila from seeded tap water by filtration and centrifugation. Appl. Environ. Microbiol. 1995, 61, 1805-1809. [CrossRef] [PubMed]

12. Ta, A.C.; Stout, J.E.; Yu, V.L.; Wagener, M.M. Comparison of culture methods for monitoring Legionella species in hospital potable water systems and recommendations for standardization of such methods. J. Clin. Microbiol. 1995, 33, 2118-2123. [CrossRef]

13. Brenner, K.P.; Rankin, C.C. New screening test to determine the acceptability of 0.45 -micron membrane filters for analysis of water. Appl. Environ. Microbiol. 1990, 56, 54-64. [CrossRef]

14. Smith, L.; Carroll, K.; Mottice, S. Comparison of membrane filters for recovery of legionellae from water samples. Appl. Environ. Microbiol. 1993, 59, 344-346. [CrossRef] [PubMed]

15. Mossel, D.A. Food microbiology: An authentic academic discipline with substantial potential benefits for science and society. J. Assoc. Anal. Chem. 1991, 74, 1-13. [CrossRef]

16. Wadowsky, R.M.; Yee, R.B. Glycine-containing selective medium for isolation of Legionellaceae from environmental specimens. Appl. Environ. Microbiol. 1981, 42, 768-772. [CrossRef] [PubMed]

17. Edelstein, P.H. Comparative study of selective media for isolation of Legionella pneumophila from potable water. J. Clin. Microbiol. 1982, 16, 697-699. [CrossRef] [PubMed]

18. Hammer, B.K.; Swanson, M.S. Co-ordination of legionella pneumophila virulence with entry into stationary phase by ppGpp. Mol. Microbiol. 1999, 33, 721-731. [CrossRef] [PubMed]

19. Miyamoto, H.; Yamamoto, H.; Arima, K.; Fujii, J.; Maruta, K.; Izu, K.; Shiomori, T.; Yoshida, S. Development of a new seminested PCR method for detection of Legionella species and its application to surveillance of legionellae in hospital cooling tower water. Appl. Environ. Microbiol. 1997, 63, 2489-2494. [CrossRef] [PubMed]

20. International Organization for Standardization. ISO 11133: 2014 Microbiology of Food, Animal Feed and Water-Preparation, Production, Storage and Performance Testing of Culture Media; International Organization for Standardization: Geneva, Switzerland, 2014.

21. Edelstein, P.H.; Edelstein, M.A. Comparison of different agars used in the formulation of buffered charcoal yeast extract medium. J. Clin. Microbiol. 1991, 29, 190-191. [CrossRef] [PubMed] 
22. Deming, W.E.; Stephan, F.F. On a Least Squares Adjustment of a Sampled Frequency Table When the Expected Marginal Totals are Known. Ann. Math. Stat. 1940, 11, 427-444. [CrossRef]

23. Altham, P.M.E.; Ferrie, J.P. Comparing Contingency Tables Tools for Analyzing Data from Two Groups Cross-Classified by Two Characteristics. Hist. Methods J. Quant. Interdiscip. Hist. 2007, 40, 3-16. [CrossRef]

24. Mosteller, F. Association and Estimation in Contingency Tables. J. Am. Stat. Assoc. 1968, 63, 1-28. [CrossRef]

25. R Development Core Team. A Language and Environment for Statistical Computing; R Foundation for Statistical Computing: Vienna, Austria, 2019.

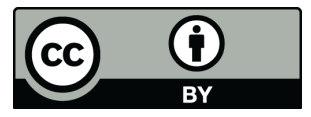

(C) 2020 by the authors. Licensee MDPI, Basel, Switzerland. This article is an open access article distributed under the terms and conditions of the Creative Commons Attribution (CC BY) license (http://creativecommons.org/licenses/by/4.0/). 